\title{
On-the-fly determination of active region centers in adaptive-partitioning QM/MM
}

\author{
Zeng-hui Yang*, $\dagger$ \\ Microsystem and Terahertz Research Center, China Academy of Engineering Physics, Chengdu, \\ China 610200, and Institute of Electronic Engineering, China Academy of Engineering Physics, \\ Mianyang, China 621000 \\ E-mail: yangzenghui@mtrc.ac.cn
}

\begin{abstract}
Quantum mechanics/molecular mechanics (QM/MM) methods partition the system into active and environmental regions and treat them with different levels of theory, achieving accuracy and efficiency at the same time. Adaptive-partitioning (AP) QM/MM methods allow on-the-fly changes to the QM/MM partitioning of the system. Many of the available energy-based AP-QM/MM method$\mathrm{s}$ partition the system according to distances to pre-chosen centers of active regions. For such APQM/MM methods, I develop an adaptive-center (AC) method that allows on-the-fly determination of the centers of active regions according to general geometrical or potential-related criteria, extending the range of application of energy-based AP-QM/MM methods to systems where active regions may occur or vanish during the simulation.
\end{abstract}

\section{Introduction}

Quantum mechanics/molecular mechanics (QM/MM) methods ${ }^{1-7}$ partition the system into regions around active sites (active regions) and the environment.Atoms in the active regions and the environment are treated with higher-level (QM) and lower-level (MM) theories respectively, and

\footnotetext{
${ }^{*}$ To whom correspondence should be addressed

${ }^{\dagger}$ Microsystem and Terahertz Research Center, China Academy of Engineering Physics, Chengdu, China 610200

Institute of Electronic Engineering, China Academy of Engineering Physics, Mianyang, China 621000
}

the two parts of the system are coupled together, ${ }^{2,8-11}$ so that the key physics of the system can be correctly described in an inexpensive simulation. Adaptive-partitioning (AP) ${ }^{6,7,12-21}$ methods enhances QM/MM by allowing the partition of the atoms to change during a molecular dynamics (MD) simulation, and they have been successfully applied to a variety of systems including solids, solutions, and biological systems. ${ }^{22-30}$

AP-QM/MM methods introduce buffer region$\mathrm{s}$ in between active regions and the environmen$\mathrm{t}$, and atoms in buffer regions (buffer atoms) are treated in both the QM and MM calculations. Force-based AP-QM/MM methods ${ }^{12,14,31,32}$ mixes the QM and MM forces to yield forces on buffer atoms, while energy-based AP-QM/MM method$\mathrm{s}^{15,16,18,19}$ mixes QM and MM potential energies of subsystems with different number of buffer atoms to obtain the total potential energy. Forcebased methods are generally more efficient but are not energy-conserving, while energy-based methods have a continuous potential energy surface (PES) and can be used in microcanonical (NVE) MD simulations.

Although there are many different ways for APQM/MM methods to determine the partition of the system, a large number of AP-QM/MM methods carry out the partition according to distances to centers of active regions. ${ }^{12-16,18,19,31,32}$ These centers can be determined on-the-fly as well as the partition in force-based AP-QM/MM methods, ${ }^{14,23,33-35}$ but the existing energy-based AP$\mathrm{QM} / \mathrm{MM}$ methods require the centers to be chosen 
beforehand. This limits the range of application of energy-based methods, since these centers may occur, vanish, or move to unpredictable places during many processes, such as in the displacement cascade, ${ }^{36}$ diffusion of self-interstitials, ${ }^{37}$ cracking of solid, ${ }^{23}$ growth of pores, ${ }^{38}$ catalytic reactions with poisoning and reactivation, ${ }^{39}$ enzymatic reactions with cofactors, ${ }^{40}$ and so on. A method that can determine centers of active regions on the fly while keeping the PES continuous would enable the use of AP-QM/MM on such applications.

In this paper, I present an adaptive-center (AC) AP method for energy-based AP-QM/MM methods, allowing the centers of active regions to be determined on the fly. Any atomic property that is continuous and solely depends on the geometry of the system can be used as the criterion. The ACAP method is compatible with all QM/MM methods that partition the system by distance to centers, and is applicable with any choice of QM and MM methods. I demonstrate the AC-AP method with two examples using different properties as criteria.

\section{Method}

\subsection{Adaptive partitioning with fixed and adaptive centers}

In the following, I use QM and MM regions as synonyms of the active and environmental region$\mathrm{s}$, even for MM/MM simulations. There are a variety of schemes that partition the system into QM, buffer and MM regions, ${ }^{15-19,21,41-44}$ and the most common choice is to partition by the distance to active region centers. ${ }^{15-19}$ The centers can be either an arbitrary point or fixed on an atom ${ }^{15}$ or on a pseudoatom. ${ }^{45}$ In this paper, I only discuss the case where the centers are fixed on certain atoms.

A center $\zeta$ has an associated radius of the QM region $R_{\zeta}^{\mathrm{QM}}$ and an associated thickness of the buffer region $W_{\zeta}^{\text {buf }}$. The QM region of $\zeta$ is the spherical region centered at $\zeta$ with radius $R_{\zeta}^{\mathrm{QM}}$, and the buffer region of $\zeta$ is the concentric spherical-shell region outside the QM region with thickness $W_{\zeta}^{\text {buf }}$. The region outside the QM and buffer region of any center is the MM region. Throughout this paper, I denote atoms in the
QM, buffer, or MM regions as QM, buffer, or M$\mathrm{M}$ atoms, and I denote the atoms chosen as active region centers as center atoms.

Each atom is assigned a weight, which represent its percentage of QM character. For an atom $\alpha$, one way to define its QM character with respect to center $\zeta$ is 15

$\tilde{\lambda}_{\alpha, \zeta}= \begin{cases}1 & R_{\alpha, \zeta} \leq R_{\zeta}^{\mathrm{QM}} \\ 1-\frac{R_{\alpha, \zeta}-R_{\zeta}^{\mathrm{QM}}}{W_{\zeta}^{\mathrm{buf}}} & R_{\alpha, \zeta} \in\left(R_{\zeta}^{\mathrm{QM}}, R_{\zeta}^{\mathrm{QM}}+W_{\zeta}^{\mathrm{buf}}\right), \\ 0 & R_{\alpha, \zeta} \geq R_{\zeta}^{\mathrm{QM}}+W_{\zeta}^{\mathrm{buf}}\end{cases}$

where $R_{\alpha, \zeta}=\left|\vec{R}_{\alpha}-\vec{R}_{\zeta}\right|$ is the distance between atom $\alpha$ and center $\zeta$. As atom $\alpha$ moves from the $\mathrm{QM}$ region of center $\zeta$ to the MM region, its QM character goes from 1 to 0 continuously. To avoid possible problems due to the discontinuous derivative of Eq. (1), the weight with respect to center $\zeta$ can be defined by applying a smoothing function ${ }^{15}$ to Eq. (1):

$$
\lambda_{\alpha, \zeta}=10 \tilde{\lambda}_{\alpha, \zeta}^{3}-15 \tilde{\lambda}_{\alpha, \zeta}^{4}+6 \tilde{\lambda}_{\alpha, \zeta}^{5}
$$

The QM and buffer regions belonging to different centers may overlap, and the total weight of atom $\alpha$ is given by 15

$$
\lambda_{\alpha}=1-\prod_{\zeta}^{\text {Centers }}\left(1-\lambda_{\alpha, \zeta}\right) .
$$

Fig. 1 illustrates the partition of the bulk Si system by distances to centers.

The choice of active region centers are held fixed in existing energy-based AP-QM/MM methods. I describe in the following a new partition scheme which allows the centers to occur or vanish during the simulation. I denote these two types of partition schemes as fixed-center (FC) and adaptivecenter (AC) throughout this paper. The PES of FCAP would be discontinuous if the centers occur or vanish during the simulation, since the weights of the center atoms are always 1 , and $R_{\zeta}^{\mathrm{QM}}$ and $W_{\zeta}^{\text {buf }}$ are fixed. In the AC-AP method described in the following, I deal with the occurring or vanishing of a center by allowing the weight of a center atom to be smaller than 1 , and allowing variable $R_{\zeta}^{\mathrm{QM}}$.

I choose the centers on-the-fly according to a cri- 


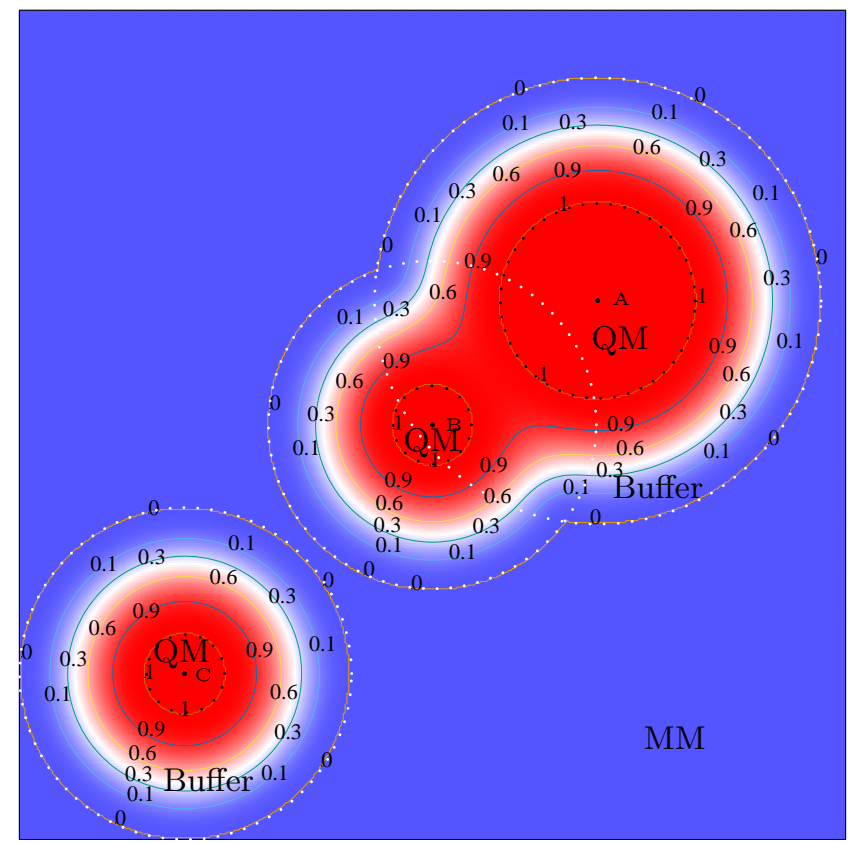

Figure 1: Illustration of the partition by distance scheme. The example has $3 \mathrm{QM}$ centers, and the buffer regions of center A and B overlap with each other. The color coding shows the weight of Eq. (3), with red being $\lambda=1$, white being $\lambda=0.5$ and blue being $\lambda=0$. The contours also show the weight. The black and white dotted circles marks out $R^{\mathrm{QM}}$ and $R^{\mathrm{QM}}+W^{\text {buf }}$ of each center. terion property, which can be any atomic property that varies continuously with respect to the geometry. Without loss of generality, I denote the criterion property of atom $\alpha$ as $\xi_{\alpha}$. There are four parameters for the AC-AP method that need to be set before the simulation: $\xi_{\min }^{\mathrm{semi}}, \xi_{\min }^{\mathrm{QM}}, \xi_{\max }^{\mathrm{QM}}$ and $R_{\max }^{\mathrm{QM}}$, which satisfy $\xi_{\min }^{\mathrm{semi}}<\xi_{\min }^{\mathrm{QM}}<\xi_{\max }^{\mathrm{QM}}$ and $R_{\max }^{\mathrm{QM}}>0$. An atom $\zeta$ is considered as an active region center if $\xi_{\zeta}>\xi_{\mathrm{min}}^{\mathrm{QM}}$, and I want its associated $R_{\zeta}^{\mathrm{QM}}$ to grow from 0 to the maximum $R_{\max }^{\mathrm{QM}}$ as $\xi_{\zeta}$ grows from $\xi_{\min }^{\mathrm{QM}}$ to $\xi_{\max }^{\mathrm{QM}}$. When $\xi_{\zeta}=\xi_{\min }^{\mathrm{QM}}$, center $\zeta$ would have no associated QM region, and its associated buffer region would become spherical with radius $W_{\zeta}^{\text {buf }}$.

I introduce semi-centers to ensure a continuous PES when active regions occur or vanish, which correspond to $\xi_{\zeta} \in\left[\xi_{\min }^{\mathrm{semi}}, \xi_{\mathrm{min}}^{\mathrm{QM}}\right)$. As $\xi_{\zeta}$ grows from $\xi_{\mathrm{min}}^{\text {semi }}$ to $\xi_{\mathrm{min}}^{\mathrm{QM}}$, the radius of the associated buffer region should grow from 0 to $W_{\zeta}^{\text {buf }}$, and the maximum $\lambda_{\alpha, \zeta}$ should grow from 0 to 1 . All these goals are achieved by defining $R_{\zeta}^{\mathrm{QM}}$ as a function of $\xi_{\zeta}$ as the following:

$$
\begin{aligned}
& R_{\zeta}^{\mathrm{QM}}=R_{\max }^{\mathrm{QM}}\left\{\theta\left(\xi_{\zeta}-\xi_{\max }^{\mathrm{QM}}\right)+\theta\left(\xi_{\max }^{\mathrm{QM}}-\xi_{\zeta}\right)\right. \\
\times & {\left.\left[\theta\left(\xi_{\zeta}-\xi_{\min }^{\mathrm{semi}}\right) f\left(\xi_{\zeta}\right)+\theta\left(\xi_{\min }^{\mathrm{semi}}-\xi_{\zeta}\right) f\left(\xi_{\min }^{\mathrm{semi}}\right)\right]\right\}, }
\end{aligned}
$$

where $\theta$ is the Heaviside step function, and $f$ is a monotonically increasing smoothing function that satisfies $f\left(\xi_{\min }^{\mathrm{QM}}\right)=0$ and $f\left(\xi_{\max }^{\mathrm{QM}}\right)=1$. The functional form of $f$ is in Appendix A.

Eq. (4) allows $R_{\zeta}^{\mathrm{QM}}$ to be negative. For a semicenter, its radius of the associated buffer region is $R_{\zeta}^{\mathrm{QM}}+W_{\zeta}^{\text {buf }}$, which should vanish when $\xi_{\zeta}=$ $\xi_{\min }$. $W^{\text {buf }}$ for all centers is therefore determined by

$$
W^{\mathrm{buf}}=-\left.R_{\zeta}^{\mathrm{QM}}\right|_{\xi_{\zeta}=\xi_{\min }^{\text {semi }}},
$$

unlike in FC-AP methods where $W^{\text {buf }}$ can be chosen freely as a parameter.

Fig. 2 shows how $R_{\zeta}^{\mathrm{QM}}$ changes with $\zeta$. The values of the curves at $\xi=0$ are equal to $-W^{\text {buf }}$ according to Eq. (5), which depends on all 4 partition parameters. I have $W^{\text {buf }}=0$ in the special case of $\xi_{\mathrm{min}}^{\mathrm{semi}}=\xi_{\mathrm{min}}^{\mathrm{QM}}$, which correspond to the 'abrup- 

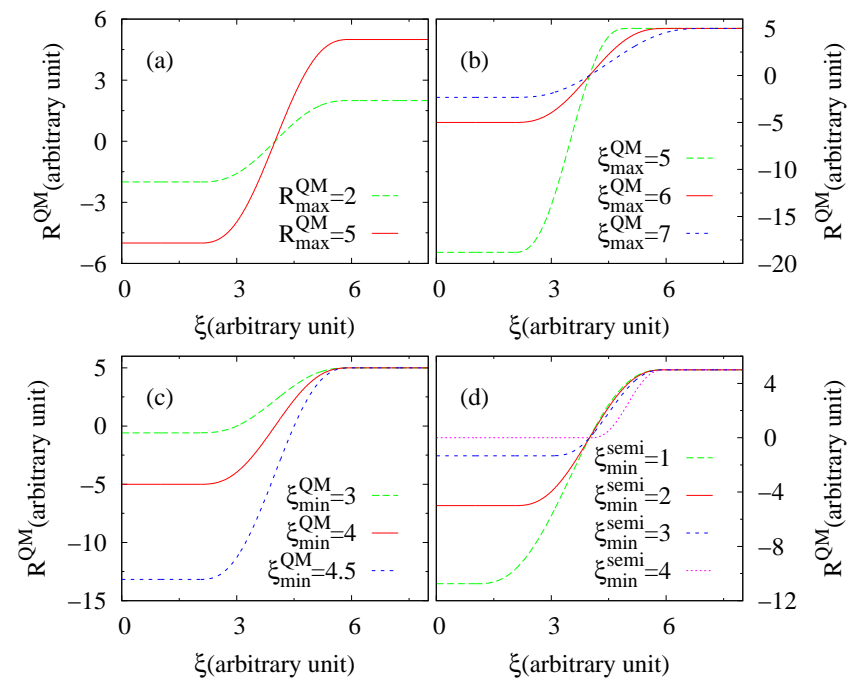

Figure 2: $R_{\zeta}^{\mathrm{QM}}$ versus $\xi_{\zeta}$ curves with different sets of partition parameters. Panel (a) is plotted with $\xi_{\min }^{\mathrm{semi}}=2, \xi_{\min }^{\mathrm{QM}}=4, \xi_{\max }^{\mathrm{QM}}=6$. Panel (b) is plotted with $R_{\max }^{\mathrm{QM}}=5, \xi_{\mathrm{min}}^{\mathrm{semi}}=2, \xi_{\mathrm{min}}^{\mathrm{QM}}=4$. Panel (c) is plotted with $R_{\max }^{\mathrm{QM}}=5, \xi_{\min }^{\mathrm{semi}}=2, \xi_{\max }^{\mathrm{QM}}=6$. Panel (d) is plotted with $R_{\max }^{\mathrm{QM}}=5, \xi_{\min }^{\mathrm{QM}}=4, \xi_{\max }^{\mathrm{QM}}=6$.

t' 5,6 AP-QM/MM calculation with discontinuous PES.

Eqs. (1), (2), and (3) remain the same in the AC-AP method. Fig. 3 illustrates $\lambda_{\alpha, \zeta}$ of Eq. (2). The ranges of $R_{\alpha, \zeta}$ where $\lambda_{\alpha, \zeta}=1$ and $\lambda_{\alpha, \zeta} \in(0,1)$ correspond to the QM and buffer regions of $\zeta$, respectively. For $\xi_{\zeta}<\xi_{\min }^{\text {semi }}$, the curve is 0 for $R \geq 0$, indicating that $\zeta$ is not an active region center. In this case, the curve has no influence on both $\lambda_{\zeta}$ and $\lambda_{\alpha}$ according to Eq. (3). For $\xi \in\left[\xi_{\min }^{\text {semi }}, \xi_{\text {min }}^{\mathrm{QM}}\right)$, the curve is above 0 in the region $R_{\alpha, \zeta} \in\left[0, R_{\zeta}^{\mathrm{QM}}+W^{\text {buf }}\right)$, indicating that $\zeta$ is a semi-center with a spherical buffer region. For $\xi \geq \xi_{\mathrm{min}}^{\mathrm{QM}}$, the curve is 1 in the region $\left[0, R_{\zeta}^{\mathrm{QM}}\right]$ and in between 0 and 1 in the region $\left[R_{\zeta}^{\mathrm{QM}}, R_{\zeta}^{\mathrm{QM}}+W^{\text {buf }}\right]$, indicating that $\zeta$ is a center with a spherical QM region and a spherical-shell shaped buffer region. The values of the curves at $R=0$ are the maximum weights with respect to center $\zeta$, and the weight of a semi-center with respect to itself is below 1 . As $\xi_{\zeta}$ increase from smaller than $\xi_{\min }^{\text {semi }}$ to larger than $\xi_{\max }^{\mathrm{QM}}$, the atom $\zeta$ changes from regular atom to semi-center and then to center smoothly. Active region centers as well as their QM and buffer regions occur or vanish smoothly during the AC-AP simulation.

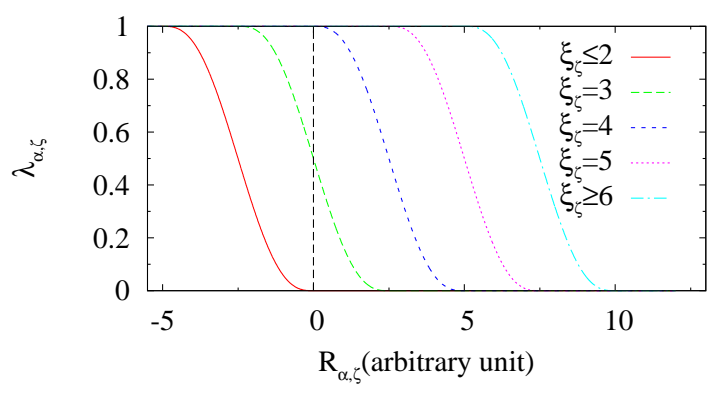

Figure 3: Weight of atom $\alpha$ with respect to center $\zeta$ as in Eq. (2) in the AC-AP method. I set $R_{\max }^{\mathrm{QM}}=2, \xi_{\min }^{\mathrm{semi}}=0.2, \xi_{\min }^{\mathrm{QM}}=0.6$, and $\xi_{\max }^{\mathrm{QM}}=1$ for Fig. 3, which are all in arbitrary units. Only the $R \geq 0$ parts of the curves are meaningful in the calculation. The curves at $R_{\alpha, \zeta}=0$ show the weight of the center with respect to itself.

I demonstrate that the partition changes continuously when centers occur/vanish with Fig. 4. Atom B is inside the QM region of center A in Fig. 4(a)(b), and is inside the buffer region of center A in Fig. 4(c)(d). I compare the case in which $\xi_{B} \in\left[\xi_{\min }^{\mathrm{semi}}, \xi_{\mathrm{min}}^{\mathrm{QM}}\right)[$ Fig. $4(\mathrm{a})(\mathrm{c})]$ with the case in which $\xi_{B}>\xi_{\min }^{\mathrm{QM}}$ [Fig. 4(b)(d)]. For Fig. 4(a)(c), $R_{B}^{\mathrm{QM}}$ is smaller than 0 , and there is no associated QM region for semi-center $B$. The white dotted circles in Fig. 4(a)(c) shows the radius of the buffer region of $\mathrm{B}$, which is smaller than $W^{\text {buf }}$. For Fig. 4(b)(d), $R^{\mathrm{QM}}$ of center $\mathrm{B}$ is greater than $0 \mathrm{~s}-$ ince $\xi_{B}>\xi_{\mathrm{min}}^{\mathrm{QM}}$, and the thickness of the buffer region equals $W^{\text {buf }}$. B is a semi-center in Fig. 4(a), but it is a QM atom with $\lambda_{B}=1$ according to Eq. (3), despite $\lambda_{B, B}$ of Eq. (2) is below 1 .

The PES of AP-QM/MM is a mixture of the PESs of the QM method and of the MM method, with the weights of Eq. (3) as mixing parameters. The dependence of the PES on the weights leads to artificial transition forces in the form of

$$
\vec{F}_{\alpha}^{\text {trans }}=-\sum_{\beta} \frac{\partial E}{\partial \lambda_{\beta}} \nabla_{\vec{R}_{\alpha}} \lambda_{\beta},
$$

which signifies the difference between the QM and MM methods. These forces are needed for ener- 


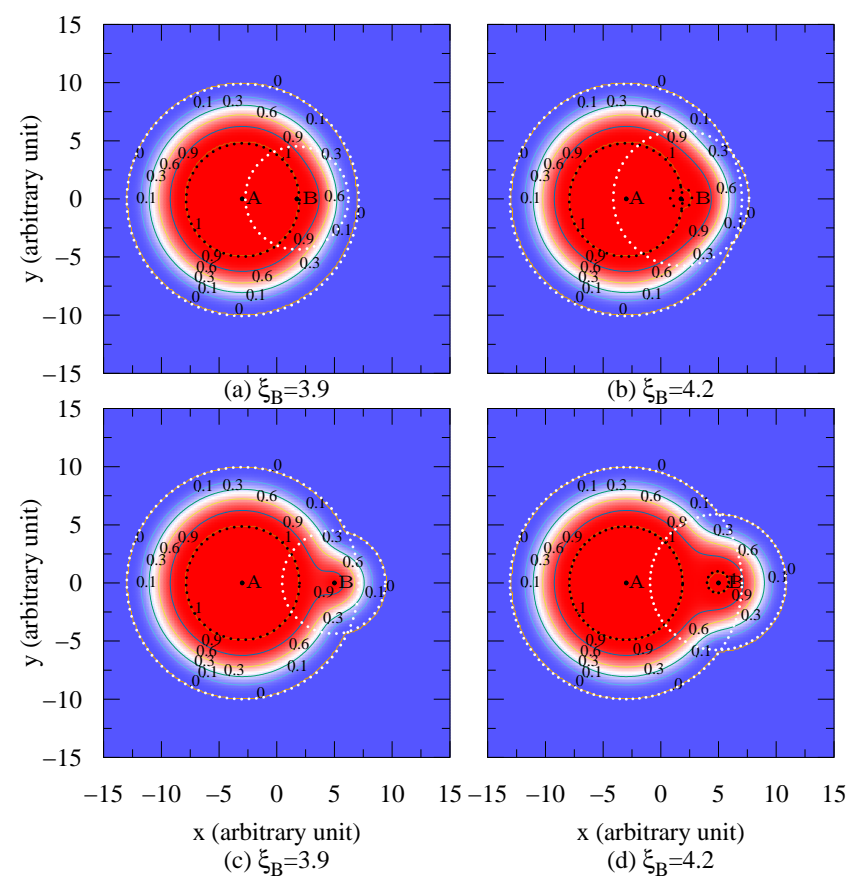

Figure 4: Illustration of the change in partition and weights as a new semi-center/center occurs. The partition parameters are $R_{\max }^{\mathrm{QM}}=5, \xi_{\min }^{\mathrm{semi}}=2$, $\xi_{\min }^{\mathrm{QM}}=4, \xi_{\max }^{\mathrm{QM}}=6 . W^{\text {buf }}=5$ according to Eq. (5).

gy conservation, though it has been found that the transition forces may lead to geometry distortions. ${ }^{16,45,46}$ In the AC-AP method, the weight of an atom not only depends on the distances between the atom and its associated QM centers, but also depends on the values of the criterion property of the QM centers, so the transition forces would contain an additional term:

$\vec{F}_{\alpha}^{\text {trans }}=-\sum_{\beta}\left[\frac{\partial E}{\partial \lambda_{\beta}}\left(\nabla_{\vec{R}_{\alpha}} \lambda_{\beta}+\sum_{\gamma} \frac{\partial \lambda_{\beta}}{\partial \xi_{\gamma}} \nabla_{\vec{R}_{\alpha}} \xi_{\gamma}\right)\right]$

Most of the existing AP-QM/MM methods carry out multiple $\mathrm{QM} / \mathrm{MM}$ calculations per time step. ${ }^{15,16,18}$ In these methods, part of the buffer atoms are included in the QM calculations, and the others are included in the MM calculations. AP-QM/MM method with only one QM and M$\mathrm{M}$ calculations per time step has been proposed as well, ${ }^{19,47}$ which involves calculations with scaled interactions. Since the partition scheme only defines the QM, buffer and MM regions and does not interfere with how this information is used in $\mathrm{QM} / \mathrm{MM}$ calculations, the AC-AP method is compatible with all the existing partition-by-distance
AP-QM/MM methods.

The AC-AP method is not without flaws, however. First, the size of the buffer region ( $\left.W^{\text {buf }}\right)$ is fixed by the partition parameters and cannot be set freely, which may lead to suboptimal situations since the computational cost usually increases very fast with the number of buffer atoms. Second, the QM calculations may encounter numerical instabilities due to nearly isolated atoms, which may be present during the occurring or vanishing of active regions. Third, the criterion property itself need to be continuous with respect to atomic positions, so some widely used properties (such as the common neighbor analysis, ${ }^{48,49}$ central symmetry parameter, ${ }^{50}$ and so on) cannot be directly employed as the criterion. These problems mostly do not exist for force-based AP-QM/MM such as the LOTF method, and only arise in energy-based AP-QM/MM due to the requirement of a continuous PES.

\section{Results}

I demonstrate the AC-AP method with two examples in bulk $\mathrm{Si}$, one being the displacement cascade process, ${ }^{36}$ the other being the diffusion of the self-interstitial. ${ }^{37}$ These two examples are chosen to showcase the AC-AP method with different properties as the criterion: I use a potential-related atomic property as the criterion in the first example, and a geometrical property as the criterion in the second.

The purpose of these MD simulations is only to validate the AC-AP method, and they are not supposed to be proper studies of the corresponding processes. The actual $\mathrm{QM}$ or $\mathrm{MM}$ method used in the AC-AP-QM/MM simulation is irrelevant to the AC-AP method. I therefore simply carry out AC-AP-MM/MM simulations to minimize the computational cost, instead of using a proper QM method as the higher-level method. I keep the notation "QM regions" and "QM atoms" for these AC-AP-MM/MM simulations for consistency, and I continue to refer to the MM potentials employed for "QM regions" and "MM regions" as the "higher-level" and "lower-level" methods respectively. For simplicity, I use the modSISPA $^{19,47}$ AP-QM/MM algorithm in the exam- 
ples, but any energy-based AP-QM/MM method that partition by distance would be compatible as well.

\subsection{Displacement cascade in Silicon}

During the irradiation of solids with energetic particles, the incident particles deposit part of their energy to the material, lead to dislodgement of atoms and displacement cascades. ${ }^{36,51,52}$ For semiconductor devices, displacement cascades may happen during the ion implantation process ${ }^{53-55}$ in the fabrication, and in radiation environments such as the space, nuclear reactors and particle accelerators. ${ }^{52,56,57}$ Displacement cascades are difficult to observe experimentally as they happen fast inside the material, ${ }^{36}$ so atomistic simulations ${ }^{57,58}$ are essential for their study.

\subsubsection{The virial contribution to the per-atom stress as AC-AP criterion}

The structures of the collision cascades generated by fast-moving atoms are far from the equilibrium structure, ${ }^{36}$ and the fast-moving atoms disturb the local electronic structure strongly, leading to the electronic stopping power. ${ }^{51,59}$ It is therefore meaningful to treat the regions around the fastmoving atoms with a higher-level method, so that both the the electronic effect and forces on atoms can be accurately described.

The speed of an atom cannot be used as a criterion here, since the Hamiltonian of the system would no longer equal to the total energy, ${ }^{60}$ and the usual velocity-Verlet time-integration ${ }^{61,62}$ would be unapplicable. As an alternative, I find the pre-atom virial contribution to the stress tensor a good indicator of the active sites, since it identifies the local structure distortions caused by collision of atoms. The criterion of this example is therefore calculated as the following.

The per-atom stress tensor of atom $\alpha\left(\stackrel{\leftrightarrow}{\sigma}_{\alpha}\right)$ is given by ${ }^{63-66}$

$$
\begin{aligned}
\stackrel{\leftrightarrow}{\sigma}_{\alpha} & =\frac{1}{V_{\alpha}} S_{\alpha} \\
& =\frac{1}{V_{\alpha}}\left(\stackrel{\leftrightarrow}{S}_{\mathrm{K}, \alpha}+\stackrel{\leftrightarrow}{S}_{\mathrm{V}, \alpha}\right),
\end{aligned}
$$

where $V_{\alpha}$ is the atomic volume of $\alpha$, and the ki- netic contribution $\stackrel{\leftrightarrow}{S}_{\mathrm{K}, \alpha}$ and virial contribution $\stackrel{\leftrightarrow}{S}_{\mathrm{V}, \alpha}$ are

$$
\begin{aligned}
& \stackrel{\leftrightarrow}{S}_{\mathrm{K}, \alpha}=m_{\alpha} \vec{v}_{\alpha} \otimes \vec{v}_{\alpha} \\
& \stackrel{\leftrightarrow}{S}_{\mathrm{V}, \alpha}=\vec{r}_{\alpha} \otimes \vec{F}_{\alpha}
\end{aligned}
$$

with $\otimes$ representing tensorial direct product, $V_{\alpha}$ being the volume of atom $\alpha, m_{\alpha}, \vec{r}_{\alpha}, \vec{v}_{\alpha}, \vec{F}_{\alpha}$ being the mass, position, velocity and force of $\alpha$ respectively. Since $V_{\alpha}$ is not a well-defined quantity, I work with $\stackrel{\leftrightarrow}{S}_{\alpha}$ instead of $\stackrel{\leftrightarrow}{\sigma}_{\alpha}$ in the following.

$\stackrel{\leftrightarrow}{S}_{\mathrm{K}, \alpha}$ depends on the velocity, so using the ful1 per-atom stress tensor as the criterion property would lead to a PES that depends on both the geometry of the system and the velocities of the atoms. The Hamiltonian of such a system is no longer equal to the total energy, ${ }^{60}$ so both the equation of motion (EOM) and time-integration algorithm are different from those of regular MD. To avoid such complications, $\stackrel{\leftrightarrow}{S}_{\mathrm{V}, \alpha}$ instead of $\stackrel{\leftrightarrow}{S}_{\alpha}$ is a more favorable choice as the criterion property for AC-AP.

$S_{\alpha}$ depends on both the geometry and the force on atom $\alpha$. The forces on QM or MM atoms in AP-QM/MM are determined completely by the geometry of the system, but the forces on buffer atoms also depend on the weights of Eq. (3). The weights depend on the partition of the system, which in turn is determined by the values of the criterion property of atoms. To avoid the extra computational cost associated with the selfconsistent determination of $\stackrel{\leftrightarrow}{S}_{\alpha}$ when using it as the criterion property, I choose to simply evaluate $\stackrel{\leftrightarrow}{S}_{\alpha}$ with another MM potential, so that the forces can be obtained directly from the geometry. For simplicity, I evaluate $\stackrel{\leftrightarrow}{S}_{\alpha}$ with the shifted LennardJones (LJ) potential

$$
E^{\mathrm{LJ}}=\sum_{\alpha, \beta>\alpha} E_{\alpha \beta}^{\mathrm{LJ}}
$$

and the pairwise LJ interaction energy between atom $\alpha$ and $\beta$ is

$E_{\alpha \beta}^{\mathrm{LJ}}= \begin{cases}4 \varepsilon_{\alpha \beta}\left[\left(\frac{\sigma_{\alpha \beta}}{R_{\alpha \beta}}\right)^{12}-\left(\frac{\sigma_{\alpha \beta}}{R_{\alpha \beta}}\right)^{6}\right]+s_{\alpha \beta} & R_{\alpha \beta} \leq R^{\mathrm{c}} \\ 0 & R_{\alpha \beta}>R^{\mathrm{c}}\end{cases}$ 
where $R_{\alpha \beta}=\left|\vec{R}_{\alpha}-\vec{R}_{\beta}\right|$ is the distance between atom $\alpha$ and $\beta, R^{\mathrm{c}}$ is a cutoff distance, $\varepsilon_{\alpha \beta}$ and $\sigma_{\alpha \beta}$ are LJ parameters and $s_{\alpha \beta}$ is a shift that ensures $E_{\alpha \beta}^{\mathrm{LJ}}=0$ when $R_{\alpha \beta}=R^{\mathrm{c}}$.

In tests with the DD process in bulk $\mathrm{Si}$, I find that the LJ $\stackrel{\leftrightarrow}{S}_{\mathrm{V}}$ changes rapidly over many orders of magnitude. In order to achieve a smooth transition as QM centers occur and vanish, I use the following criterion property for the following displacement cascade simulation:

$$
\xi_{\alpha}=\log _{10}\left|S_{\mathrm{V}, \alpha}^{x x}+S_{\mathrm{V}, \alpha}^{y y}+S_{\mathrm{V}, \alpha}^{z z}\right| .
$$

\subsubsection{Displacement cascade with AC-AP}

I carry out microcanonical (NVE) MD simulations of the displacement cascade of bulk Si with a $15 \times 15 \times 20$ supercell (36000 atoms), starting from the state equilibrated at $300 \mathrm{~K}$ in the NVT ensemble. One Si atom is chosen as the primary knock-on atom (PKA) before the simulations, and its velocity is set to $5 \mathrm{keV}$ along the positive $z$ axis. I use the following partition parameters for AC$\mathrm{AP}: R_{\max }^{\mathrm{QM}}=4 \AA, \xi_{\text {min }}^{\mathrm{semi}}=8, \xi_{\min }^{\mathrm{QM}}=9, \xi_{\max }^{\mathrm{QM}}=10$. The simulations are run for $0.05 \mathrm{ps}$ with a $10^{-6} \mathrm{p}$ $\mathrm{s}$ time step. The purpose of the examples is only to validate the AC-AP method, as a proper simulation of the displacement cascade would involve a much larger supercell and much longer simulation time. ${ }^{36,58,67-70}$

I use the NVE ensemble to check energy conservation, but I also notice that proper simulations for displacement cascades ${ }^{36,58,68-70}$ are carried out in the NVE ensemble as well, only with an additional surrounding temperature control layer. I omit the temperature control layer since I find its effec$t$ on the examples being very small, probably due to the simulation time being too short for the local heating effect of the cascade to propagate to the boundary.

I carry out an AC-AP-MM/MM simulation using the Tersoff potential ${ }^{71}$ modified with the Ziegler-Biersack-Littmark (ZBL) short-range interaction ${ }^{72}$ as the "higher-level" method, and the charge optimized many-body $(\mathrm{COMB})^{73-75}$ potential as the "lower-level" method. The AC-AP $\mathrm{MM} / \mathrm{MM}$ calculation with these potentials is denoted as (Tersoff/ZBL)/COMB in the following.
All the simulations have the same initial state prepared with Tersoff/ZBL, so that the results can be compared directly. I do not assign charges to atoms for $\mathrm{COMB}$, since charge equilibration $(\mathrm{QE}-$ q) ${ }^{73,76}$ methods are incompatible with the modSISPA ${ }^{19,47}$ algorithm.

I use the LJ parameters of the OpenKIM project $^{77-80}$ to evaluate the criterion. I implement the AC-AP method and the mod-SISPA algorithm in the LAMMPS ${ }^{81,82}$ code. I use the OVITO $^{83}$ software for the visualization of the MD results.

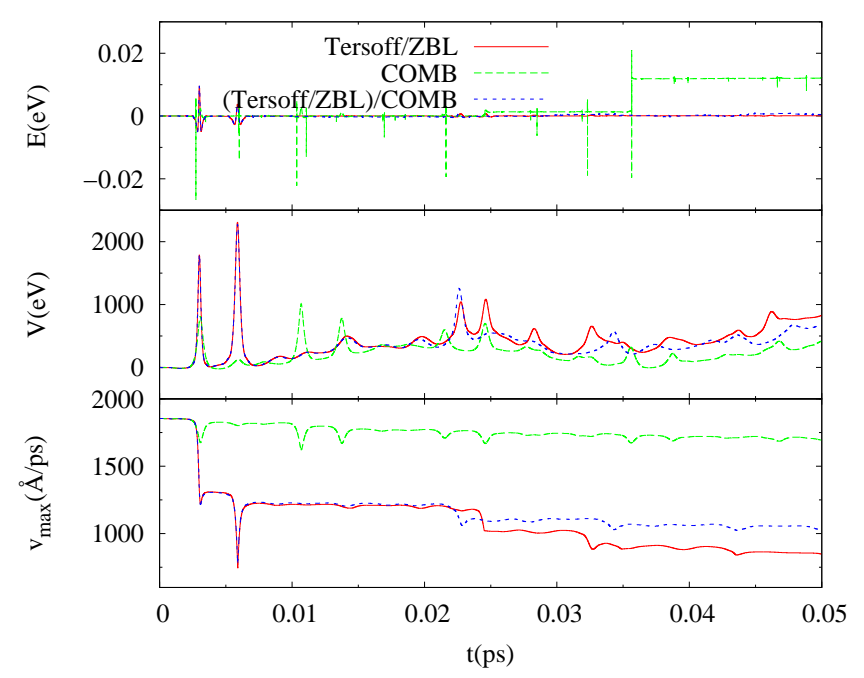

Figure 5: Curves of the total energy, the potential energy and the maximum speed of atoms for MD simulations of the displacement cascade in bulk Si. The energy curves are shifted and aligned at $t=0$ for easier comparison.

The upper panel of Fig. 5 plots the total energies with respect to the simulation time. The small variations in the total energies are due to numerical errors in the time integration, since the magnitudes of these variations decrease with the size of the time step in tests. The size of the numerical variation in the total energy of AC-AP $(0.01 \mathrm{eV})$ is similar to those of the MM methods (COMB: $0.03 \mathrm{eV}$, Tersoff/ZBL: $0.01 \mathrm{eV}$ ). These total energy variations happen when atoms collide, as indicated by large changes in the potential energy (middle panel of Fig. 5). A variable time step ${ }^{36,84}$ can effectively remove this numerical artifact.

As atoms collide, LJ forces of the colliding atoms change rapidly, so are the values of the ACAP criterion property according to Eqs. (10) and (13). This is reflected by the change of the num- 


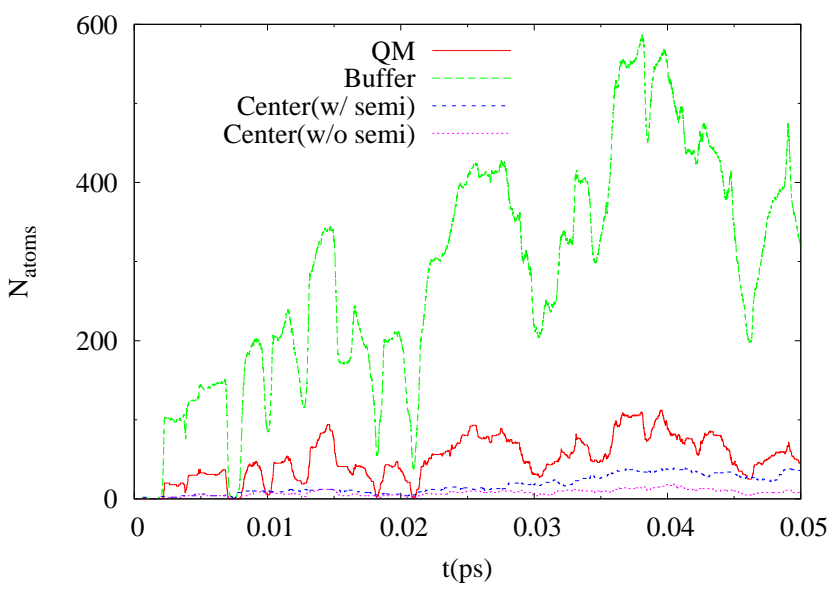

Figure 6: Numbers of "QM" atoms, buffer atoms and active region centers (both with and without semi-centers) plotted against the simulation time. The setup of the simulation is the same as in Fig. 5.

ber of active region centers plotted in Fig. 6. Since $R^{\mathrm{QM}}$ is different for each center, the number of "QM" and buffer atoms do not necessarily follow the same trend as the number of centers. Fig. 6 shows that the partition of the system undergoes rapid changes during the simulation, with both active region centers occuring/vanishing and "QM"/buffer regions enlarging/shrinking. Despite this, the total energy of the AC-AP simulation remain conserved, demonstrating the validity of the method.

\begin{tabular}{|c|c|c|}
\hline COMB • & Tersoff/ZBL & $\begin{array}{l}\text { AC-AP } \\
\text { (Tersoff/ZBL)/COMB }\end{array}$ \\
$\vdots$ & & \\
$\vdots$ & $\vdots$ & $\vdots$ \\
$\vdots$ & $\ddots$ & \\
\hline
\end{tabular}

Figure 7: Front view ( $x z$ plane) of the WignerSeitz defect analysis ${ }^{83}$ of snapshots of MD simulations at $0.05 \mathrm{ps}$, with red representing interstitial defects and blue representing vacancies.

The AC-AP curves of the potential energy and the speed of the PKA (middle and lower panel of Fig. 5) closely follows the corresponding curves of the "higher-level" Tersoff/ZBL for the first half of the simulations, showing that the AC-AP method correctly uses the "higher-level" interaction to describe the collision processes. The deviation of the curves in the second half of the simulations shows the influence of the "lower-level" COMB potential in the MM/MM simulation, and suggests that Eq. (13) may not be the perfect choice of the criterion for the simulation of displacement cascades. Nevertheless, Fig. 7 shows that the overall shape of the AC-AP cascade is still very close to that of the "higher-level" Tersoff/ZBL, where cascades branch early and sub-cascades develop; in the "lower-level" COMB simulation, the cascade develops along an almost straight path.

\begin{tabular}{|c|c|c|}
\hline$t=0$ & $t=0.01 \mathrm{ps}$ & $t=0.02 \mathrm{ps}$ \\
& & \\
\hline$t=0.03 \mathrm{ps}$ & $t=0.04 \mathrm{ps}$ & $t=0.05 \mathrm{ps}$ \\
$\vdots$ & & \\
\hline & $\vdots$ & $\vdots$ \\
\hline
\end{tabular}

Figure 8: Front view snapshots of the AC-AP simulation with (Tersoff/ZBL)/COMB, showing the criterion property $\xi$ of Eq. (13). Only atoms with $\xi>\xi_{\text {min }}^{\text {semi }}$ are shown. The color coding represents $\xi$, with red being $\xi=\xi_{\max }^{\mathrm{QM}}$, white being $\xi=\xi_{\min }^{\mathrm{QM}}$, and blue being $\xi=\xi_{\min }^{\text {semi }}$.

For a more quantitative comparison, I list several properties of the displacement cascade simulations $^{36}$ in Table 1, including the number of interstitials $\left(N_{\text {int }}\right)$, number of atoms displaced by more than half the nearest neighbor distance $\left(N_{\text {displ }}\right)$, the mean square displacement of all atoms (MSD), the root-mean-square distance of interstitials to the interstitial center of mass $\left(\left\langle d R_{\text {int }}\right\rangle\right)$, the root-meansquare differences of the $\mathrm{x}, \mathrm{y}$ and $\mathrm{z}$ coordinates between the interstitials and the interstitial center of mass ( $\left\langle d x_{\text {int }}\right\rangle,\left\langle d y_{\text {int }}\right\rangle$, and $\left.\left\langle d z_{\text {int }}\right\rangle\right)$, and the $\max$ speed of all atoms $\left(v_{\max }\right)$. All the properties calculated according to the geometry of the system at 
the end of the simulations ( $0.05 \mathrm{ps})$. These values can be compared directly since all the simulation$\mathrm{s}$ start from exactly the same initial state. Aside from $\left\langle d R_{\text {int }}\right\rangle$, the values of the properties of ACAP with (Tersoff/ZBL)/COMB are always in between those of the two MM simulations as expected. Most of the AC-AP values of the properties are closer to the "higher-level" Tersoff/ZBL values than to the "lower-level" COMB values, showing the validity of Eq. (13) as the criterion in identifying the active sites. $\left\langle d R_{\text {int }}\right\rangle$ apparently does not follow this trend, but this is due to $\left\langle d R_{\text {int }}\right\rangle$ not distinguishing directions. $\left\langle d x_{\text {int }}\right\rangle,\left\langle d y_{\text {int }}\right\rangle$, and $\left\langle d z_{\text {int }}\right\rangle$ are better quantities to describe the spread of the cascade in this case.

To provide a clear picture of the inner work of the AC-AP method during the simulation, I show the snapshots of the AC-AP (Tersoff/ZBL)/COMB simulation in Figs. 8, 9, and 10. Corresponding animations are available as supplemental material$\mathrm{s}^{85}$ of this paper. Fig. 8 shows the active region centers determined by the AC-AP method, and the values of the criterion property $\xi$ is indicated by the color coding.

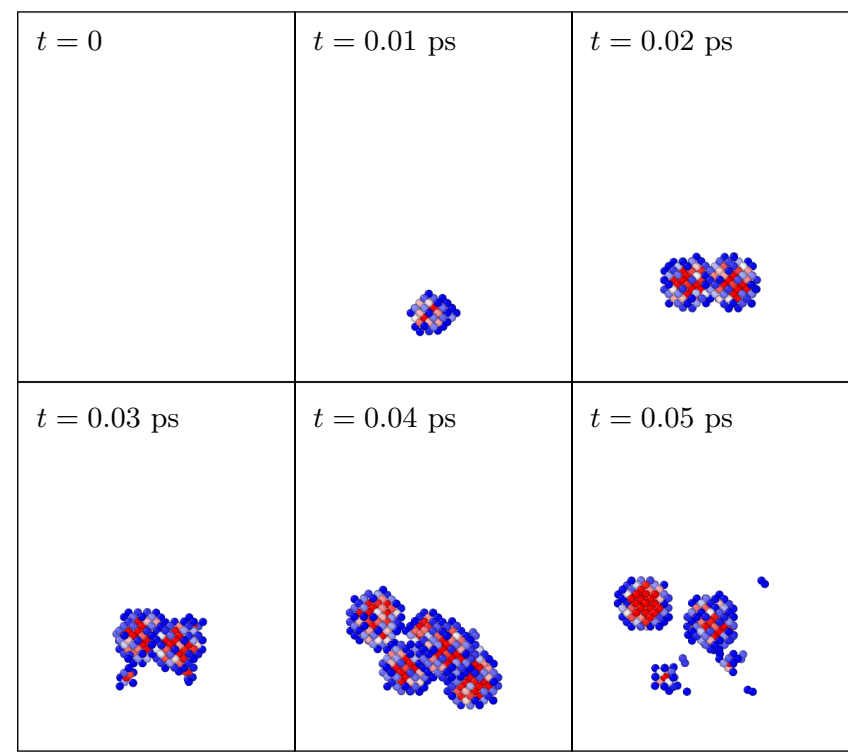

Figure 9: Front view snapshots of the AC-AP simulation with (Tersoff/ZBL)/COMB, showing the weights of atoms. Only atoms with $\lambda>0$ are shown. The color coding represents the weight$\mathrm{s}$, with red being $\lambda=1$, white being $\lambda=0.5$ and blue being $\lambda=0$.

I find that the centers are generated at places where atoms are very close to each other. As a result, centers always appear in pairs or in cluster$\mathrm{s}$ and never appear alone. As the clustered centers are very close to each other, their "QM" and buffer regions mostly overlap, so the clustered centers usually do not lead to an increase in the computational cost. This is verified with Fig. 9 which shows the "QM" and buffer atoms. Fig. 9 clearly shows that the "QM" and buffer regions of different centers have different radii. In the $t=0.05$ ps panel of Fig. 9, there are centers that just occur/almost vanish with $\xi$ close to $\xi_{\min }^{\text {semi }}$. The buffer regions of these centers have very small radii and does not contain any other atoms. When using a QM method as the higher-level method, these nearly isolated atoms may lead to stability problem$\mathrm{s}$, and this is a flaw of the AC-AP method of this work. In tests with the density-functional tightbinding (DFTB) method, ${ }^{86-88}$ I find that the problems caused by nearly isolated atoms can be solved by using fractional occupation numbers according to the Fermi-Dirac distribution at a finite temperature, but there is no guarantee that this would always work.

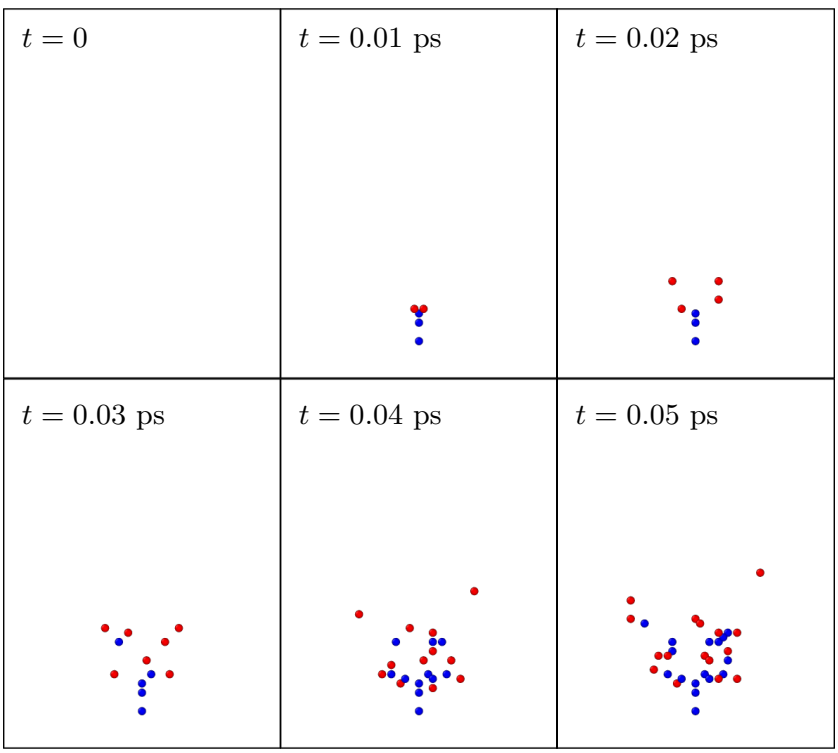

Figure 10: Front view of the Wigner-Seitz defect analysis ${ }^{83}$ of snapshots of the AC-AP simulation with (Tersoff/ZBL)/COMB. The color coding is the same as in Fig. 7.

Fig. 10 shows the development of the displace cascade in AC-AP. Since the simulation time is very small, annealing of defects is not observed. By comparing Figs. 8 and 10, I find that the development of active region centers follow that of 
Table 1: Various properties of the displacement cascade MD simulations at $t=0.05 \mathrm{ps}$.

\begin{tabular}{ccccccccc}
\hline \hline Method & $N_{\text {int }}$ & $N_{\text {displ }}$ & MSD $\left(\AA^{2}\right)$ & $\left\langle d R_{\text {int }}\right\rangle(\AA)$ & $\left\langle d x_{\text {int }}\right\rangle(\AA)$ & $\left\langle d y_{\text {int }}\right\rangle(\AA)$ & $\left\langle d z_{\text {int }}\right\rangle(\AA)$ & $v_{\max }(\AA / \mathrm{ps})$ \\
\hline COMB & 10 & 21 & 0.2494 & 26.28 & 2.579 & 7.220 & 25.14 & 1694 \\
Tersoff/ZBL & 24 & 51 & 0.1660 & 25.30 & 12.95 & 19.94 & 8.628 & 847.0 \\
AC-AP & 17 & 41 & 0.1820 & 23.34 & 11.02 & 18.60 & 8.79 & 1028 \\
\hline \hline
\end{tabular}

sub-cascades, that new active region centers occur at the places around which new defects are generated, and old active region centers vanish as old defects stabilize. In an AC-AP QM/MM simulation, the generation of defects would be treated with a QM method, so that the electronic effect$\mathrm{s}^{51,59}$ could be included to yield a more accurate result.

\subsection{Diffusion of self-interstitial in Sil- icon}

The electrical properties of semiconductor materials can be modified significantly by defects and impurities. ${ }^{89}$ The diffusion process of defects and impurities in Silicon has been thoroughly studied $^{37}$ to provide a better understanding of the performance drift of Si-based devices. The diffusivity of the various defects and impurities are key to the simulation of the technological processes. ${ }^{90}$ The diffusion of the self-defects in bulk $\mathrm{Si}$ has been used in the validation of the 'Learnon-the-fly' 14,34 (LOTF) force-based AP-QM/MM method, where the QM region is centered on the defect. I perform similar simulations for the diffusion of the self-interstitial in bulk Si with the ACAP method, and choose the criterion property so that the interstitial can be identified as the center of the active region.

\subsubsection{Generalized coordination number as the AC-AP criterion}

The self-interstitial atom is not always well defined. The equilibrium structure has a dumbbell shape, ${ }^{91}$ in which the two $\mathrm{Si}$ atoms of the dumbbell are equivalent. The migration process happens as one atom in the dumbbell moves to the tetrahedral interstitial site and then forms a dumbbell structure with another atom. It is therefore impossible to fix the center of the active region on an atom chosen beforehand, and an AC method has to be used for an AP-QM/MM method.

I use the coordination number $(\mathrm{CN})$ to identify the Si self-interstitial in the following simulations. The $\mathrm{CN}$ is defined as the number of nearest neighbors of an atom. Most of the atoms in bulk Si has a $\mathrm{CN}$ of 4 . I find that the $\mathrm{CNs}$ of the interstitial and some of its neighboring atoms are generally larger and can have the value of 5 or 6 . The $\mathrm{CN}$ is not the perfect choice since in some cases the interstitial atom labeled out by the Wigner-Seitz defect analysis ${ }^{83}$ has $\mathrm{CN}=4$ as well.

Since the $\mathrm{CN}$ is an integer, it does not change continuously, so it cannot be used as the criterion for the AC-AP method directly. To generalize the $\mathrm{CN}$ for its use as the criterion, I first write $\mathrm{CN}$ of an atom $\alpha$ as

$$
\mathrm{CN}_{\alpha}=\sum_{\zeta \neq \alpha} \theta\left(R_{1}-R_{\alpha, \zeta}\right)
$$

where $R_{1}$ is a cutoff radius. When the temperature is below the melting point, I can choose $R_{1}$ so that Eq. (14) is equal to the number of nearest neighbors of $\alpha$ for bulk Si without defects. I find that the self-interstitial can be identified as having a $\mathrm{CN}$ larger than 4 in most configurations.

I generalize the $\mathrm{CN}$ defined in Eq. (14) by replacing the step function with a continuous function as

$$
\begin{aligned}
& \mathrm{GCN}_{\alpha}=\sum_{\zeta \neq \alpha} \theta\left(R_{1}-R_{\alpha, \zeta}\right) \\
& +\theta\left(R_{\alpha, \zeta}-R_{1}\right) \theta\left(R_{2}-R_{\alpha, \zeta}\right) g\left(\frac{R_{\alpha, \zeta}-R_{2}}{R_{1}-R_{2}}\right),
\end{aligned}
$$

where GCN stands for generalized coordination number, $R_{2}$ is another cutoff greater than $R_{1}$, and

$$
g(x)=10 x^{3}-15 x^{4}+6 x^{5}
$$

is a smoothing function. I choose to use the same 
form as Eq. (2) for the smoothing function $g$. I use the GCN of Eq. (15) as the criterion $\xi_{\alpha}$ for the following diffusion simulation.

\subsubsection{Diffusion of self-interstitial with AC-AP}

I calculate the diffusivity of the a single selfinterstitial in bulk $\mathrm{Si}$ with a $6 \times 6 \times 6$ supercel1 (1729 atoms) in the NVE ensemble, following the practice of Tang et al. ${ }^{37}$ Since the exact trajectory of the self-interstitial is not as important as in the displacement cascade simulation, I do not need to start from the same initial state for all the simulations. Instead, I start from equilibrated initial states with optimized lattice constants of the corresponding MD potential, so that the temperatures of the NVE simulations oscillates around that of the initial states, which ensures the reliability of the diffusivity calculations. I use the following parameters for AC-AP: $R_{1}=3 \AA, R_{2}=3.5 \AA, R_{\max }^{\mathrm{QM}}=4 \AA$, $\xi_{\min }^{\mathrm{semi}}=6, \xi_{\min }^{\mathrm{QM}}=6.35, \xi_{\max }^{\mathrm{QM}}=6.7$. I simulate the system for $200 \mathrm{ps}$ with a time step of $5 \times 10^{-5} \mathrm{p}$ $\mathrm{s}$, and the diffusivities are obtained by fitting the MSD of the last 150 ps. I use the same potentials and softwares as in Sec. 3.1.2.

I show snapshots of the AC-AP simulation with (Tersoff/ZBL)/COMB in Fig. 11. There are always two interstitial atoms in Fig. 11(a), which agrees with the dumbbell structure ${ }^{91}$ of the selfinterstitial in Silicon. Fig. 11(b) shows that the GCN may not be the ideal choice of the criterion: it only identifies one interstital atom in many situations, and unrelated atoms may be chosen as active region centers; furthermore, during the migration of the interstitial to another site, GCN may fail to identify any atom as the active region center. Despite these problems, I choose GCN as the criterion property of this example instead of more sophisticated methods ${ }^{92}$ for its simplicity in implementation, which suffices for the demonstration of the AC-AP method.

Fig. 12 is the Arrhenius plot of the diffusivity of the self-interstitial in Silicon. To demonstrate the effect of the AC-AP parameters on the result, I also plot in Fig. 12 the AC-AP result with an alternative set of parameters $\left(\xi_{\min }^{\mathrm{semi}}=5, \xi_{\min }^{\mathrm{QM}}=6, \xi_{\max }^{\mathrm{QM}}=7\right)$. The slopes and intercepts of the interpolation lines determine the migration energy and the diffusivity prefactor. ${ }^{37}$ Since the GCN may fail to identify
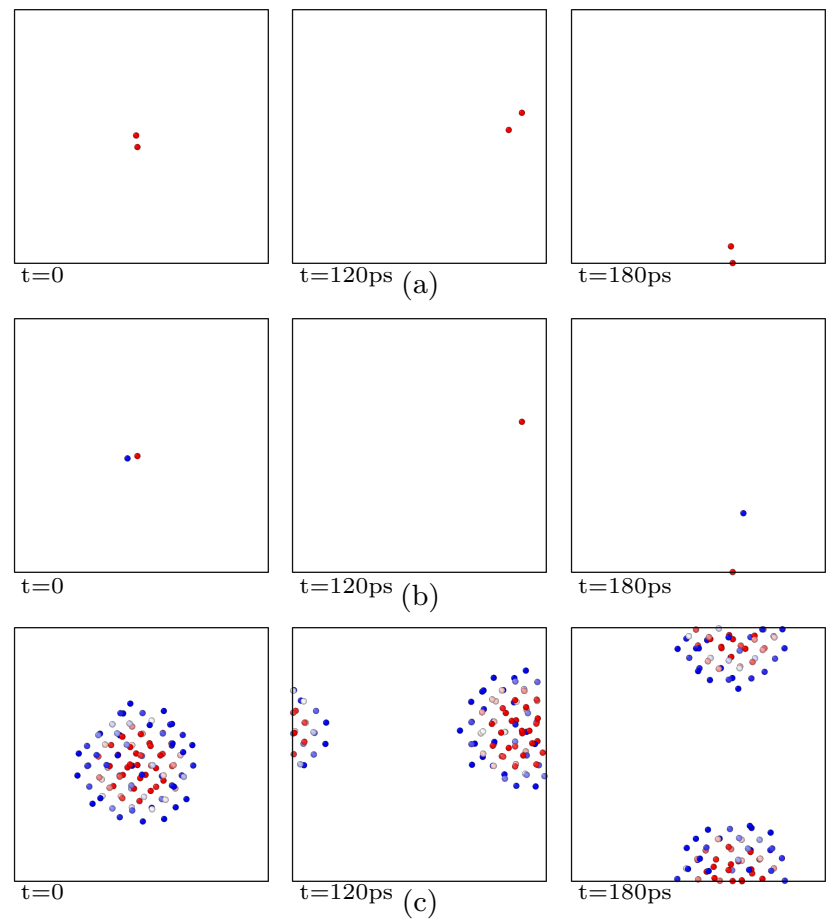

Figure 11: Front view of the snapshots of the (Tersoff/ZBL)/COMB simulation of the diffusion of the self-interstitial in bulk Si, showing (a) the position of the self-interstitial obtained by the WignerSeitz defect analysis, ${ }^{83}$ (b) the value of the criterion property, and (c) the weights of atoms. The average temperature of the NVE simulation is 2052K. The color coding is the same as Figs. 8, 9 , and 10 .

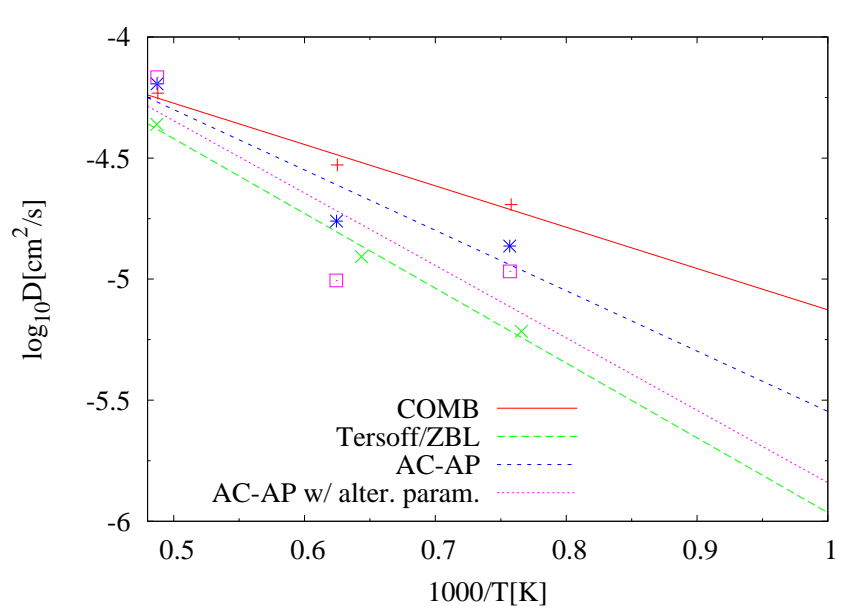

Figure 12: Temperature-dependent diffusivities for a single self-interstitial in bulk Si. The line labeled 'AC-AP w/ alter. param.' shows the data of the AC-AP (Tersoff/ZBL)/COMB simulation with an alternative set of parameters $\left(\xi_{\mathrm{min}}^{\mathrm{semi}}=5, \xi_{\mathrm{min}}^{\mathrm{QM}}=\right.$ $6, \xi_{\max }^{\mathrm{QM}}=7$ ). 
any atom as the active region center in certain circumstances, the evolution of the system are completely determined by the "lower-level" COMB potential at times. Due to this problem of the GC$\mathrm{N}$, the error in the AC-AP diffusivity (obtained by fitting MSD data) is larger, which is evident in Fig. 12.

This problem of the GCN as criterion is also consistent with the two AC-AP lines in Fig. 12 lying in between the interpolation lines of the two component methods of the MM/MM. A better criterion that is able to identify the interstitial atom at all circumstances would yield an interpolation line closer to the line of the "higher-level" Tersoff/ZBL potential. This can be checked with a smaller $\xi_{\min }^{\text {semi }}$ : the interstitial atom would be more likely to be identified as active region center, so the migration process would be less affected by the "lower-level" COMB potential. The AC-AP line with the alternative set of parameters in Fig. 12 demonstrates this point.

\section{Conclusion}

In this paper, I generalize energy-based AP$\mathrm{QM} / \mathrm{MM}$ methods by presenting an AC-AP method that is able to determine active region centers on-the-fly, enabling easy application of energy-based AP-QM/MM methods on systems where active regions may occur or vanish, or on systems where it is impossible to fix the active region centers on atoms chosen beforehand. The AC-AP method replaces hand-picking active region centers by choosing an atomic property as the criterion. The only restriction on the criterion property is that it has to be fully determined by the geometry of the system, so that the dynamics of the system remain Lagrangian. Only four partition parameters $\left(R_{\max }^{\mathrm{QM}}, \xi_{\min }^{\mathrm{semi}}, \xi_{\min }^{\mathrm{QM}}, \xi_{\max }^{\mathrm{QM}}\right)$ need to be set before the simulation. Since the AC-AP method only determine the partition of the system and the weights of atoms, it is compatible with all energy-based AP-QM/MM methods that partition the system according to distances to active region centers, and it can be easily incorporated into existing simulation codes.

The AC-AP method ensures a continuous PES by allowing the radius of the QM and buffer re- gions associated with active region cores to change with the values of the criterion property. I treat the occurring and vanishing of active regions by introducing semi-centers which has only associated buffer region but no QM region. As an active region occur in the simulation, the radius of the buffer region of the semi-center grows from 0 to $W^{\text {buf }}$ of Eq. (5), and the maximum atomic weight with respect to the semi-center grows from 0 to 1 ; the reverse is true for the vanishing of an active region.

I demonstrate the AC-AP method with two examples in bulk Si using different properties as criteria. Since the main purpose of these examples is to validate the AC-AP method instead of proper studies of the corresponding problems, I use $\mathrm{MM} / \mathrm{MM}$ simulations with AC-AP for simplicity. Total energies are conserved for AC-AP simulations in the NVE ensemble. Values of typical properties of the AC-AP MM/MM simulations are found to be in between the corresponding values of the two MM simulations. The partition parameters have a large impact on AC-AP results, and can be adjusted for the AC-AP results to be closer to those of the higher-level method.

\section{Acknowledgements}

Acknowledgement The author is supported by Science Challenge Project, No. TZ2016003, and by the National Natural Science Foundation of China grant No. 11804314.

\section{A Smoothing function of the $\mathrm{QM}$ region radius}

$f(\xi)$ in Eq. (4) is a monotonically increasing continuous function that satisfies $f\left(\xi_{\max }^{\mathrm{QM}}\right)=1$ and $f\left(\xi_{\min }^{\mathrm{QM}}\right)=0$. In practice, I only need to evaluate $f(\xi)$ for $\xi \in\left[\xi_{\min }^{\text {semi }}, \xi_{\max }^{\mathrm{QM}}\right]$. I obtain a smoothly changing $f(\xi)$ as the following. Define $\tau^{\prime}(\tau)$ as

$$
\tau^{\prime}(\tau)=\frac{\tau-\tau_{\mathrm{min}}^{\mathrm{semi}}}{1-\tau_{\mathrm{min}}^{\mathrm{semi}}},
$$


where $\tau(\xi)=\left(\xi-\xi_{\min }^{\mathrm{QM}}\right) /\left(\xi_{\max }^{\mathrm{QM}}-\xi_{\min }^{\mathrm{QM}}\right)$, and $\tau_{\min }^{\text {semi }}=\tau\left(\xi_{\text {min }}^{\text {semi }}\right) . \quad$ I write $f(\xi)$ as $f(\xi)=$ $g\left(\tau^{\prime}(\tau(\xi))\right)$, and $g$ needs to satisfy the following conditions so that the first and second order derivatives of Eq. (4) are continuous:

$$
\begin{gathered}
g\left(\tau^{\prime}(0)\right)=0,\left.\quad \underset{g(1)=1,}{\left.\frac{d g\left(\tau^{\prime}\right)}{d \tau^{\prime}}\right|_{\tau^{\prime}=0}=0,} \quad \frac{d g\left(\tau^{\prime}\right)}{d \tau^{\prime}}\right|_{\tau^{\prime}=1}=0, \\
\left.\frac{d^{2} g\left(\tau^{\prime}\right)}{d \tau^{\prime 2}}\right|_{\tau^{\prime}=0}=0,\left.\quad \frac{d^{2} g\left(\tau^{\prime}\right)}{d \tau^{\prime 2}}\right|_{\tau^{\prime}=1}=0 .
\end{gathered}
$$

Assuming $g\left(\tau^{\prime}\right)$ has the following functional form

$$
g\left(\tau^{\prime}\right)=a \tau^{\prime 5}+b \tau^{\prime 4}+c \tau^{\prime 3}+d \tau^{\prime 2}+e \tau^{\prime}+f,
$$

the parameters satisfying Eq. (18) are

$$
\begin{aligned}
& a=\frac{6}{\left[1-\tau^{\prime}(0)\right]^{3}\left[1+3 \tau^{\prime}(0)+6 \tau^{\prime}(0)^{2}\right]}, \\
& b=\frac{15}{\left[\tau^{\prime}(0)-1\right]^{3}\left[1+3 \tau^{\prime}(0)+6 \tau^{\prime}(0)^{2}\right]} \\
& c=\frac{10}{\left[1-\tau^{\prime}(0)\right]^{3}\left[1+3 \tau^{\prime}(0)+6 \tau^{\prime}(0)^{2}\right]}, \\
& d=0, \quad e=0, \\
& f=\frac{\tau^{\prime}(0)^{3}\left[10-15 \tau^{\prime}(0)+6 \tau^{\prime}(0)^{2}\right]}{\left[\tau^{\prime}(0)-1\right]^{3}\left[1+3 \tau^{\prime}(0)+6 \tau^{\prime}(0)^{2}\right]}
\end{aligned}
$$

\section{References}

(1) Warshel, A.; Levitt, M. J. Mol. Biol. 1976, 103, 227.

(2) Lin, H.; Truhlar, D. G. Theor. Chem. Acc. 2007, 117, 185.

(3) Senn, H. M.; Thiel, W. Angew. Chem. Int. Ed. 2009, 48, 1198.

(4) Bernstein, N.; Kermode, J. R.; Csányi, G. Rep. Prog. Phys. 2009, 72, 026501.

(5) Bulo, R. E.; Michel, C.; Fleurat-Lessard, P.; Sautet, P. J. Chem. Theory Comput. 2013, 9, 5567.

(6) Zheng, M.; Waller, M. P. WIREs Comput. Mol. Sci. 2016, 6, 369.

(7) Duster, A. W.; Wang, C.-H.; Garza, C. M.; Miller, D. E.; Lin, H. WIREs Comput. Mol. Sci. 2017, 7, e1310.
(8) Maseras, F.; Morokuma, K. J. Comput. Chem. 1995, 16, 1170.

(9) Humbel, S.; Sieber, S.; Morokuma, K. J. Chem. Phys. 1996, 105, 1959.

(10) Svensson, M.; Humbel, S.; Froese, R. D. J.; Matsubara, T.; Sieber, S.; Morokuma, K. J. Phys. Chem. 1996, 100, 19357.

(11) Bakowies, D.; Thiel, W. J. Phys. Chem. 1996, 100, 10580.

(12) Kerdcharoen, K. R., T. Liedl; Rode, B. M. Chem. Phys. 1996, 211, 313.

(13) Kerdcharoen, T.; Morokuma, K. Chem. Phys. Lett. 2002, 355, 257.

(14) Csányi, G.; Albaret, T.; Payne, M. C.; de Vita, A. Phys. Rev. Lett. 2004, 93, 175503.

(15) Heyden, A.; Lin, H.; Truhlar, D. G. J. Phys. Chem. B 2007, 111, 2231.

(16) Bulo, R. E.; Ensing, B.; Sikkema, J.; Visscher, L. J. Chem. Theory Comput. 2009, 5, 2212.

(17) Nielsen, S. O.; Bulo, R. E.; Moore, P. B.; Ensing, B. Phys. Chem. Chem. Phys. 2010, 12, 12401.

(18) Watanabe, H. C.; Kubař, T.; Elstner, M. J. Chem. Theory Comput. 2014, 10, 4242.

(19) Field, M. J. J. Chem. Theory Comput. 2017, $13,2342$.

(20) Watanabe, H. Molecules 2018, 23, 1882.

(21) Chen, H.; Liao, M.; Wang, H.; Wang, Y.; Zhang, L. Comput. Methods Appl. Mech. Eng. 2019, 354, 351.

(22) Park, K.; Götz, A. W.; Walker, R. C.; Paesani, F. J. Chem. Theory Comput. 2012, 8, 2868.

(23) Gleizer, A.; Peralta, G.; Kermode, J. R.; de Vita, A.; Sherman, D. Phys. Rev. Lett. 2014, 112, 115501. 
(24) Peguiron, A.; Ciacchi, L. C.; de Vita, A.; Kermode, J. R.; Moras, G. J. Chem. Phys. 2015, 142, 064116.

(25) Watanabe, H. C.; Banno, M.; Sakurai, M. Phys. Chem. Chem. Phys. 2016, 18, 7318.

(26) Watanabe, H. C.; Kubillus, M.; Kubař, T.; Stach, R.; Mizaikoff, B.; Ishikita, H. Phys. Chem. Chem. Phys. 2017, 19, 17985.

(27) Boereboom, J. M.; Fleurat-Lessard, P.; Bulo, R. E. J. Chem. Theory Comput. 2018, 14, 1841.

(28) Duster, A.; Garza, C.; Lin, H. Methods Enzymol. 2016, 577, 341.

(29) Duster, A. W.; Garza, C. M.; Aydintug, B. O.; Negussie, M. B.; Lin, H. J. Chem. Theory Comput. 2019, 15, 892.

(30) Duster, A. W.; Lin, H. J. Chem. Theory Comput. 2019, 15, 5794.

(31) Rode, B. M.; Hofer, T. S.; Randolf, B. R.; Schwenk, C. F.; Xenides, D.; Vchirawongkwin, V. Theor. Chem. Acc. 2006, 115, 77.

(32) Bernstein, N.; Várnai, C.; Solt, I.; Winfield, S. A.; Payne, M. C.; Simon, I.; Fuxreiter, M.; Csányi, G. Phys. Chem. Chem. Phys. 2012, 14, 646.

(33) Payne, M. C.; Csányi, G.; Albaret, T.; de Vita, A. ChemPhysChem 2005, 6, 1731.

(34) Csányi, G.; Albaret, T.; Moras, G.; Payne, M. C.; de Vita, A. J. Phys.: Condens. Matter 2005, 17, R691.

(35) Moras, G.; Csanyi, G.; Payne, M. C.; de Vita, A. Physica B 2006, 376-377, 936.

(36) Nordlund, K.; Ghaly, M.; Averback, R. S.; Caturla, M.; Diaz de la Rubia, T.; Tarus, J. Phys. Rev. B 1998, 57, 7556.

(37) Tang, M.; Colombo, L.; Zhu, J.; Diaz de la Rubia, T. Phys. Rev. B 1997, 55, 14279.

(38) Nakano, A.; Kalia, R. K.; Vashishta, P. Phys. Rev. Lett. 1994, 73, 2336.
(39) Kumar, M.; Hammond, G. B.; Xu, B. Org. Lett. 2014, 16, 3452.

(40) Chenault, H. K.; Simon, E. S.; Whitesides, G. M. Biotechnol. Genet. Eng. Rev. 1988, 6, 221.

(41) Takenaka, N.; Kitamura, Y.; Koyano, Y.; Nagaoka, M. Chem. Phys. Lett. 2012, 524, 56.

(42) Waller, M. P.; Kumbhar, S.; Yang, J. ChemPhysChem 2014, 15, 3218.

(43) Zheng, M.; Kuriappan, J. A.; Waller, M. P. Int. J. Quantum Chem. 2017, 117, 25336.

(44) Glukhova, O. E.; Savostyanov, G. V.; Slepchenkov, M. M. Procedia Materials Science 2014, 6, 256.

(45) Pezeshki, S.; Davis, C.; Heyden, A.; Lin, H. J. Chem. Theory Comput. 2014, 10, 4765.

(46) Boereboom, J. M.; Potestio, R.; Donadio, D.; Bulo, R. E. J. Chem. Theory Comput. 2016, 12,3441 .

(47) Yang, Z.-H. DOI: 10.26434/chemrxiv.11916015.

(48) Faken, D.; Jónsson, H. Comput. Mater. Sci. 1994, 2, 279.

(49) Tsuzuki, H.; Branicio, P. S.; Rino, J. P. Comput. Phys. Comm. 2007, 177, 518.

(50) Kelchner, C. L.; Plimpton, S. J.; Hamilton, J. C. Phys. Rev. B 1998, 58, 11085.

(51) Race, C. P.; Mason, D. R.; Finnis, M. W.; Foulkes, W. M. C.; Horsfield, A. P.; Sutton, A. P. Rep. Prog. Phys. 2010, 73, 116501.

(52) Srour, J. R.; Palko, J. W. Displacement damage effects in devices. 2013.

(53) Chason, E.; Picraux, S. T.; Poate, J. M.; Borland, J. O.; Current, M.; Diaz de la Rubia, T.; Eaglesham, D. J.; Holland, O. W.; Law, M. E.; Magee, C. W.; Mayer, J. W.; Melngailis, J.; Tasch, A. F. J. Appl. Phys. 1997, 81, 6513 . 
(54) Williams, J. S.; Elliman, R. G.; Ridgway, M. C.; Jagadish, C.; Ellingboe, S. L.; Goldberg, R.; Petravic, M.; Wong, W. C.; Dezhang, Z.; Nygren, E.; Svensson, B. G. Nucl. Instrum. Methods Phys. Res., Sect. B 1993, 80-81, 507.

(55) Borland, J. O.; Koelsch, R. Solid State Technol. 1993, 36, 28.

(56) Srour, J. R. IEEE Trans. Nucl. Sci. 2003, 50, 653.

(57) Myers, S. M.; Cooper, P. J.; Wampler, W. R. J. Appl. Phys. 2008, 104, 044507.

(58) Lan, M.; Yang, Z.-H.; Wang, X. Comput. Mater. Sci. 2020, 179, 109697.

(59) Keinonen, J.; Arstila, K.; Tikkanen, P. Appl. Phys. Lett. 1992, 60, 628.

(60) Thornton, S. T.; Marion, J. B. Classical dynamics of particles and systems; Brooks/Cole, 2004.

(61) Verlet, L. Phys. Rev. 1967, 159, 98.

(62) Donnelly, D.; Rogers, E. Am. J. Phys. 2005, $73,938$.

(63) Tsai, D. H. J. Chem. Phys. 1979, 70, 1375.

(64) Swenson, R. J. Amer. J. Phys. 1983, 51, 940.

(65) Subramaniyan, A. K.; Sun, C. T. Int. J. Solids Struct. 2008, 45, 4340 .

(66) Thompson, A. P.; Plimpton, S. J.; Mattson, W. J. Chem. Phys. 2009, 131, 154107.

(67) Nordlund, K.; Keinonen, J.; Kuronen, A.

(68) Gao, F.; Bacon, D. J. Philos. Mag. A 1995, 71,65 .

(69) Zhu, H.; Averback, R. S.; Nastasi, M. Philos. Mag. A 1995, 71, 735.

(70) Nordlund, K.; Ghaly, M.; Averback, R. S. J. Appl. Phys. 1998, 83, 1238.

(71) Tersoff, J. Phys. Rev. B 1988, 37, 6991.
(72) Ziegler, J. F.; Biersack, J. P.; Littmark, U. The stopping and ranges of ions in solids; Pergamon Press: New York, 1985.

(73) Yu, J.; Sinnott, S. B.; Phillpot, S. R. Phys. Rev. B 2007, 75, 085311.

(74) Shan, T.-R.; Devine, B. D.; Kemper, T. W.; Sinnott, S. B.; Phillpot, S. R. Phys. Rev. B 2010, $81,125328$.

(75) Liang, T.; Shan, T.-R.; Cheng, Y.-T.; Devine, B. D.; Noordhoek, M.; Li, Y.; Lu, Z.; Phillpot, S. R.; Sinnott, S. B. Mat. Sci. Eng. $R$ 2013, 74, 255.

(76) Rappé, A. K.; Goddard, W. A., III J. Phys. Chem. 1991, 95, 3358.

(77) Elliott, R. S. Efficient 'universal' shifted Lennard-Jones model for all KIM API supported species developed by Elliott and Akerson (2015) v003. 2018; doi:10.25950/962b4967.

(78) Elliott, R. S. Efficient multi-species LennardJones model with truncated or shifted cutoff v003. 2018; doi:10.25950/ac258694.

(79) Tadmor, E. B.; Elliott, R. S.; Sethna, J. P.; Miller, R. E.; Becker, C. A. JOM 2011, 63, 17.

(80) Elliott, R. S.; Tadmor, E. B. Knowledgebase of Interatomic Models (KIM) Application Programming Interface (API). 2011; doi:10.25950/ff8f563a.

(81) Plimpton, S. J. J. Comput. Phys. 1995, 117, 1

(82) http:// lammps.sandia.gov.

(83) Stukowski, A. Modelling Simul. Mater. Sci. Eng. 2010, 18, 015012.

(84) Smith, R.; Harrison, D. E., Jr. Computers in Physics 1989, 3, 68.

(85) Refer to the supplemental material of this paper. 
(86) Porezag, D.; Frauenheim, T.; Köhler, T.; Seifert, G.; Kaschner, R. Phys. Rev. B 1995, $51,12947$.

(87) Elstner, M.; Porezag, D.; Jungnickel, G.; Elsner, J.; Haugk, M.; Frauenheim, T.; Suhai, S.; Seifert, G. Phys. Rev. B 1998, 58, 7260 .

(88) Frauenheim, T.; Seifert, G.; Elstner, M.; Hajnal, Z.; Jungnickel, G.; Porezag, D.; Suhai, S.; Scholz, R. Phys. Stat. Sol. (b) 2000, 217, 41 .

(89) Yu, P. Y.; Cardona, M. Fundamentals of Semiconductors: Physics and Materials Properties, 4th ed.; Springer: Berlin, 2010.

(90) Sentaurus ${ }^{\mathrm{TM}}$ Process User Guide.

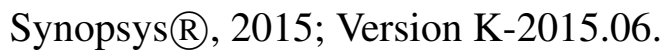

(91) Zhu, J.; Diaz de la Rubia, T.; Yang, L. H.; Mailhiot, C.; Gilmer, G. H. Phys. Rev. B 1996, 54, 4741.

(92) Li, D.; Wang, F.; Yang, Z.; Zhao, Y. Sci. China Phys. Mech. Astron. 2014, 57, 2177. 